\title{
Metallicity Gradients of Thick Disk Dwarf Stars
}

\author{
Kenneth Carrell, Yuqin Chen and Gang Zhao \\ Key Laboratory of Optical Astronomy, National Astronomical Observatories, Chinese Academy of Sciences, \\ Beijing 100012, China \\ carrell@nao.cas.cn
}

\begin{abstract}
We examine the metallicity distribution of the Galactic thick disk using F, G, and $\mathrm{K}$ dwarf stars selected from the Sloan Digital Sky Survey, Data Release 8. Using the large sample of dwarf stars with proper motions and spectroscopically determined stellar parameters, metallicity gradients in the radial direction for various heights above the Galactic plane and in the vertical direction for various radial distances from the Galaxy center have been found. In particular, we find a vertical metallicity gradient of $-0.113 \pm 0.010(-0.125 \pm 0.008)$ dex $\mathrm{kpc}^{-1}$ using an isochrone (photometric) distance determination in the range $1<|Z|<3 \mathrm{kpc}$, which is the vertical height range most consistent with the thick disk of our Galaxy. In the radial direction, we find metallicity gradients between +0.02 and $+0.03 \mathrm{dex} \mathrm{kpc}^{-1}$ for bins in the vertical direction between $1<|Z|<3 \mathrm{kpc}$. Both of these results agree with similar values determined from other populations of stars, but this is the first time a radial metallicity gradient for the thick disk has been found at these vertical heights. We are also able to separate thin and thick disk stars based on kinematic and spatial probabilities in the vertical height range where there is significant overlap of these two populations. This should aid further studies of the metallicity gradients of the disk for vertical heights lower than those studied here but above the solar neighborhood. Metallicity gradients in the thin and thick disks are important probes into possible formation scenarios for our Galaxy and a consistent picture is beginning to emerge from results using large spectroscopic surveys, such as the ones presented here.
\end{abstract}

Subject headings: galaxy: abundances - galaxy: disk

\section{Introduction}

The thick disk of our Galaxy has been extensively studied (especially in the solar neighborhood) since it was discovered by Gilmore \& Reid (1983). Photometric and spectroscopic studies have shown that it is a distinct component in a number of ways. Jurić et al. (2008) find that the stellar number density distribution of dwarf stars in the Galactic disk found using Sloan Digital Sky Survey (SDSS) data and a photometric parallax method is well fit with a two-component exponential. The thick disk component is also known to have both a rotational lag and a larger velocity dispersion than the thin disk Chiba \& Beers 2000; Soubiran et al. 2003) giving it a distinct kinematic signature. It has further been shown to be the dominant component for stars with vertical distances of $1<|Z|<3 \mathrm{kpc}$ from the Galactic plane (Allende Prieto et al. 2006).

The metallicity distribution of the thick disk is still an unresolved issue, however. Using the metallicity and kinematics of SDSS stars, Ivezić et al. (2008) are able to reproduce the results of previous works, but also caution that there are problems with describing their data using a two-component exponential. Most importantly, they suggest that the metallicity and velocity distributions can be used to describe a single disk component instead of two separate ones. This would suggest a complex nature to a single disk component and not two distinct disk components with different formations and evolutions. More re- 
cently, Bovy et al. (2012) also find that the Galactic disk can be represented by a single exponential in the radial and vertical directions that is smoothly varying as a function of age (by proxy using abundances).

This has naturally lead to several competing theories as to the formation mechanism of the thick disk and how it relates to the thin disk. For example, the thick disk could be mainly composed of accreted stars from previous mergers (Abadi et al. 2003), composed of stars formed from accreted gas from previous mergers (Brook et al. 2004), or from the internal mixing of stars and gas within the Galaxy (Schönrich \& Binney 2009). These are just a few of the various internal and external sources from which the thick disk of our Galaxy could arise. Obviously, a combination of some or all of these scenarios could also give rise to our current Galactic hierarchy. Certain well-defined properties of the thick disk should be able to distinguish between these possible scenarios and provide a clearer picture of the formation and evolution of our Galactic disk.

In the radial direction, metallicity gradients vary from $\sim 0$ dex $\mathrm{kpc}^{-1}$ (Allende Prieto et al. 2006 ) to $+0.028 \pm 0.036 \mathrm{dex} \mathrm{kpc}^{-1}$ (Nordström et al. 2004) for stars likely to be thick disk members. We note that the former uses spectroscopically derived stellar parameters of F- and G-type stars observed by SDSS in the range $1<|Z|<3 \mathrm{kpc}$ while the latter uses photometrically derived stellar parameters of nearby F- and G-type stars. Recent results from the Radial Velocity Experiment (RAVE, Steinmetz et al. 2006) fall within these two values (Ruchti et al. 2011; Coşkunoğlu et al. 2012).

Vertical metallicity gradients of the thick disk show more discrepancy. Allende Prieto et al. (2006) found no variation of metallicity as a function of vertical height. Using SDSS Red Horizontal Branch stars, Chen et al. (2011) found a gradient as high as $-0.22 \pm 0.07$ dex $\mathrm{kpc}^{-1}$ but this result most likely suffered contamination from other Galactic sources. Again, the RAVE results fall somewhere in the middle (Kordopatis et al. 2011; Ruchti et al. 2011) as do the results of Katz et al. (2011) who observed stars along two different lines of sight.

Our aim in this work is to examine both ra- dial and vertical metallicity gradients of stars associated with the thick disk of our Galaxy. We use the large sample of spectroscopically observed stars from the Sloan Extension for Galactic Understanding and Exploration (SEGUE) made available in SDSS Data Release 8 (DR8). Selecting dwarf stars in this analysis is important since it is the dominant component of the disk of our Galaxy. Furthermore, these stars have changed very little since their formation, making them an excellent probe of the condition of our Galaxy during the formation of its disk. We employ various techniques used by previous analyses in order to more easily compare results from different sets of data and using different selection criteria. The large sample of stars with reliable parameter determinations facilitates this comparison and allows us to give a more comprehensive view of the metallicity distribution of stars up to several kiloparsecs above or below the Galactic plane.

\section{Data}

Spectroscopic data from SDSS DR8 Aihara et al. 2011) was obtained from the CasJobs interface. The improvement in DR8 for high-metallicity stars is important for this work since we expect many stars to have abundances near or slightly below solar. We used the segue1_target1 flag to select F, $\mathrm{G}$, and $\mathrm{K}$ dwarfs (integer values of -2147483136 , -2147221504 , and -2147450880 , respectively). We require that the stars have clean photometry and reasonable spectroscopic parameters (wide cuts were applied to eliminate any undetermined or extreme values for the effective temperature, surface gravity, metallicity and radial velocity). This leaves us with 66,481 out of the total 88,081 stars for these three categories. Further cuts were applied to ensure a clean sample of dwarf stars with good quality observed values and are summarized in Table 1. Our final sample is $43,417 \mathrm{~F}, \mathrm{G}$, and $\mathrm{K}$ dwarf stars (slightly less than half the full sample) that have well defined photometric and spectroscopic values as well as reasonable proper motions. In Figure 1 we show the spatial distribution of our dwarf star sample using distances calculated as described below.

The most stringent cut for our dwarf sample is on the surface gravity since we want a sample free of possible turnoff or giant stars to aid 
Table 1: Selection cuts for our data.

\begin{tabular}{|c|c|c|c|}
\hline Category & SDSS Criteria & Stars & Our Criteria \\
\hline $\mathrm{F} / \mathrm{G}$ & $\begin{array}{c}g<20.2 \\
0.2<(g-r)<0.48\end{array}$ & 6939 & \multirow{3}{*}{$\begin{array}{c}12.0<g, r, i<21.0 \mathrm{mag} \\
g, r, i \text { error }<0.05 \mathrm{mag} \\
\log g>4.4 \mathrm{dex} \text { AND } \log g \text { error }<0.25 \mathrm{dex} \\
\mathrm{T}_{e f f} \text { error }<250 \mathrm{~K} \\
{[\mathrm{Fe} / \mathrm{H}] \text { error }<0.25 \mathrm{dex}} \\
\text { radial velocity error }<10 \mathrm{~km} \mathrm{~s}^{-1} \\
\text { proper motion errors }<6 \mathrm{mas} \mathrm{yr}^{-1} \\
\end{array}$} \\
\hline G dwarf & $\begin{array}{c}14.0<r<20.2 \\
0.48<(g-r)<0.55\end{array}$ & 62784 & \\
\hline K dwarf & $\begin{array}{c}14.5<r<19.0 \\
0.55<(g-r)<0.75\end{array}$ & 18358 & \\
\hline TOTALS & & 88,081 & 43,417 \\
\hline
\end{tabular}

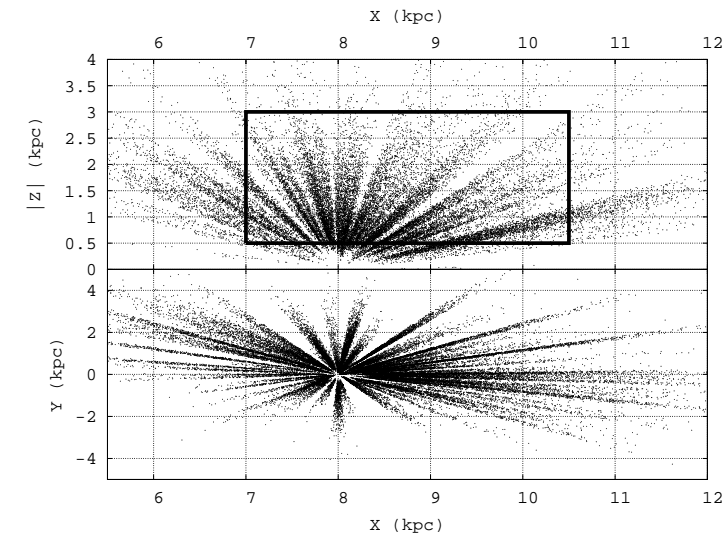

Fig. 1. - Spatial distribution of F, G, and K dwarf stars selected from SDSS DR8.

in the reliability of the distance determination. Therefore, selecting a cut on the surface gravity of 4.4 dex and requiring that the error on this value be less than 0.25 dex ensures that we will not have stars with surface gravities lower than $\sim 4.15$ dex. Furthermore, as can be seen in Figure 2. there is a noticeable change in the distribution of the metallicity as a function of surface gravity near $\log g \sim 4.4$ dex. The histogram in the upper portion of Figure 2 clearly shows that the metallicity distribution of stars with $4.1<\log g<4.4$ dex is wider and has a peak that is more metal poor than for stars with $\log g>4.4$ dex. We note that Cheng et al. (2012) concentrate on the main sequence turnoff stars that we are trying to eliminate. Also, Schlesinger et al. (2011) use G and K dwarfs in their analysis, but their surface gravity cut is $\log g>4.1$ dex. This means that their sample overlaps in surface gravities with this paper and with Cheng et al. (2012). The much steeper

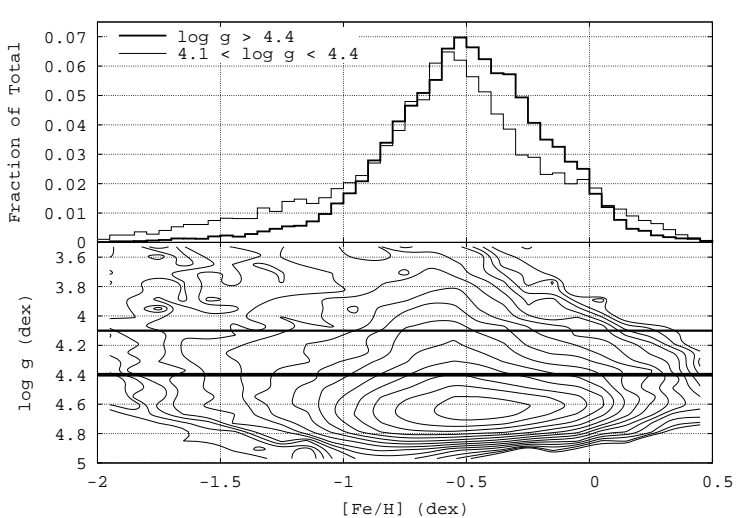

Fig. 2.- Surface gravities and metallicities for the F, G, and K dwarf stars selected from SDSS DR8. The horizontal lines in the lower plot correspond to surface gravities of 4.1 and 4.4 dex. The upper plot shows histograms of the metallicity for two surface gravity selections.

vertical metallicity gradient in Schlesinger et al. (2011) could be due to this fact as well as the modifications they apply to the metallicities provided by the SEGUE Stellar Parameter Pipeline and the weighting algorithm they use (both of which tend toward higher metallicity).

Selection bias in SDSS spectroscopic samples can be extremely difficult to determine or even estimate. Our sample comes from a relatively simple set of selection criteria so we expect the bias to be minimal. Essentially, targets were selected from a wide color range $(0.2<(g-r)<0.75)$ with more weight given to $\mathrm{G}$ type stars. Since we are not interested in a specific population, but in the dwarf stars as a whole, the number of each spectral type is not a primary concern. What is most essential is 
that the selection criteria should not influence the expected abundances of our sample stars, and a simple color cut should not introduce a significant bias in this regard.

Distance determinations were made using two methods. The first method we used was adapted from Equation 1 of Kordopatis et al. (2011), which is based on the method found in Zwitter et al. (2010). This technique uses theoretical isochrones to determine the absolute magnitude of a star with a certain set of parameters and their errors. This is, in essence, a Bayesian approach with the likelihood determined by the distance from a point on the isochrone and the prior probability determined by the mass distribution. We used Girardi et al. (2004) isochrones that were downloaded from the $\mathrm{CMD}^{1}$ web interface. The grid of isochrones has ages of 2-12 Gyr in 1 Gyr increments and is linear in $\mathrm{Z}$ (logarithmic in $[\mathrm{Fe} / \mathrm{H}]$ ) from $\mathrm{Z}=0.0001$ to 0.03 in 0.0001 increments. The range in $[\mathrm{Fe} / \mathrm{H}]$ of roughly $-2.25<[\mathrm{Fe} / \mathrm{H}]<0.2$ dex includes the expected metallicities of the dwarf stars in our sample and the reliable limits of DR8. The large age range is necessary if we want to accurately determine distances for both the (younger) thin disk and (older) thick disk stars, but since our sample is composed of only dwarf stars, this age range has little effect because all the stars in our sample should be main sequence stars.

Using the mass weighted method described above, the probability distribution for each star was determined using four observables: the spectroscopically determined effective temperature, surface gravity, and metallicity and the photometrically determined $(g-r)_{0}$ color. Individual errors on each parameter were used instead of overall errors quoted for DR8 measurements to give a better indication of the intrinsic uncertainty of the distance determination for each star. Once the probability distribution was calculated, the most probable absolute magnitude for the star was found from a weighted average and the error on this value was determined from the weighted standard deviation. Using this absolute magnitude and the measured apparent magnitude one can find the distance to the star and convert to the Galactic Cartesian system (X,Y,Z) using its position on the sky. As a further quality control

\footnotetext{
${ }^{1}$ http://stev.oapd.inaf.it/cgi-bin/cmd
}

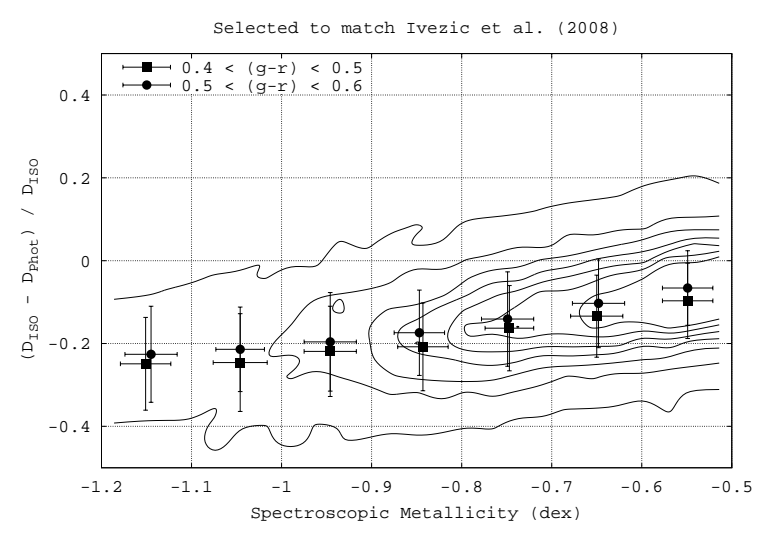

Fig. 3.- Fractional difference between distances determined using the isochrone method of Kordopatis et al. (2011) and the photometric method of Ivezić et al. (2008) as a function of metallicity for two color ranges.

of our stellar sample, we only use stars that have distance errors from the isochrone method of less than $5 \%$. This eliminates less than $0.1 \%$ of the sample, but does help identify and remove outliers and stars with inconsistent observations or parameter determinations.

The other distance determination we used came from Equation 1 of Ivezić et al. (2008). This method determines the absolute $r$ magnitude of a star using its $(g-i)$ color and metallicity. We use this method as a comparison to the isochrone method to help identify systematic errors in the distance determination. In Figure 3 we plot the fractional difference between the distances determined using the isochrone and photometric methods as a function of metallicity for two color selections. In this plot we have used the same selection criteria as that used by Ivezić et al. (2008) and determine distances using photometric metallicities derived using the equations from their work. The range of metallicities in the plot is limited to the reliable range found by Ivezić et al. (2008) for their photometric abundance determination. We find that the distances derived using the method of Ivezić et al. (2008) are systematically farther than the isochrone method of Kordopatis et al. (2011) by $\sim 10 \%$ on the high metallicity end $([\mathrm{Fe} / \mathrm{H}] \sim-$ $0.55 \mathrm{dex}$ ) and increase to $\sim 20 \%$ at lower metallicity $([\mathrm{Fe} / \mathrm{H}] \sim-1.15 \mathrm{dex})$. If, however, we use the spectroscopically determined $[\mathrm{Fe} / \mathrm{H}]$ values in- 
stead of the photometrically determined ones in the distance estimation of Ivezić et al. (2008), we find a nearly constant systematic difference of $\sim 10 \%$ for the entire metallicity range shown in Figure 3 .

Using the distance, radial velocity, and proper motion of each star, the full space motions $(\mathrm{U}, \mathrm{V}, \mathrm{W})$ were determined using the method in Johnson \& Soderblom (1987) with an updated galactic coordinate transformation. We used a modified version of the gal_uvw routine from the IDL Astronomy User's Library 2 and the solar motion values from Schönrich et al. (2010) to correct to the local standard of rest. The uncertainties in each of the velocity components are on average $\sim 20 \mathrm{~km} \mathrm{~s}^{-1}$ for our sample of stars.

\section{Analysis}

The cuts described in the previous section were applied to provide a sample of dwarf stars that could be used to isolate likely members of the disk component(s) of our Galaxy. As was described in Section 1, past studies have used various methods to select and characterize the disk. We restrict our samples to $7.0<R<10.5 \mathrm{kpc}$ since these radial distances provide us with the largest statistics and to avoid possible contamination from the bulge of the Galaxy. In the vertical direction we limit the sample to $0.5<|Z|<3.0 \mathrm{kpc}$ for determining radial metallicity gradients since there are few stars below these heights and the statistics fall rapidly above them. For vertical metallicity gradients we fit in the range $1.0<|Z|<3.0 \mathrm{kpc}$ to be more consistent with previous works and for reasons that will be discussed in Section 4 . These restrictions in radial and vertical distances are shown in Figure 1 with solid lines.

To determine the extent to which the thin disk contributes to our dwarf star sample we use the kinematic properties of the different components of the Galaxy as a discriminant. To do this, we used Equation 1 of Bensby et al. (2003) and the characteristic velocity dispersions and asymmetric drift values given in their Table 1 to determine the likelihood for each star of belonging to either the thin disk, thick disk, or halo based on its kinematics alone. The likelihood for each star of be-

\footnotetext{
${ }^{2}$ http://idlastro.gsfc.nasa.gov/
}

longing to a particular component of the Galaxy also depends on the local number density expected of that component. Since Bensby et al. (2003) use local densities for the solar neighborhood and our sample of stars is at higher vertical heights, we use the Galactic model given by Jurić et al. (2008) in column 2 of their Table 10. This is their best-fit model determined directly from the number density distribution maps found in their work. Combining the kinematic and local number density likelihoods, for each individual star we determine the likelihood of it belonging to the thin disk, thick disk, or halo. Ratios of these likelihoods were then used to find stars that are more likely to be thin disk than thick disk (i.e. TD/D $<0.10$ ) and more likely to be thick disk than thin disk or halo (i.e. $\mathrm{TD} / \mathrm{D}>10$ and $\mathrm{TD} / \mathrm{H}>10)$. These limits of being ten times more likely is in agreement with other papers employing the same technique (e.g. Coşkunoğlu et al. 2012). This helps separate stars with kinematics most obviously matching either the thin or thick disks at the heights in which both populations overlap. We note that systematics in our distance determinations can affect the local number densities for each star and can also lead to incorrect velocity components, and therefore incorrect likelihoods for belonging to one Galactic component or another. This possible issue is mitigated by the fact that we use strong cuts on the populations (i.e. being ten times more likely to be one than the other) and only use the likelihoods to separate our sample at the smallest vertical heights.

In the lower panel of Figure 4 we show that the vertical distributions for stars defined as likely thin disk and likely thick disk are very different. Thin disk stars are peaked at heights of slightly less than $0.5 \mathrm{kpc}$ and have no stars with heights larger than $\sim 0.75$ kpc. Meanwhile, the thick disk distribution is much broader and has a peak slightly higher than $1 \mathrm{kpc}$. The upper plot in Figure 4 shows that the kinematically selected thick disk sample has a peak metallicity near -0.6 dex, while the thin disk sample is peaked at a higher metallicity (near -0.2 dex). Both the vertical height distribution and the mean metallicities of the kinematically selected components are consistent with previous works. Of note is the fact that it is possible to separate the thin and thick disks in SDSS data using their kinematics and distances. Also, we see 

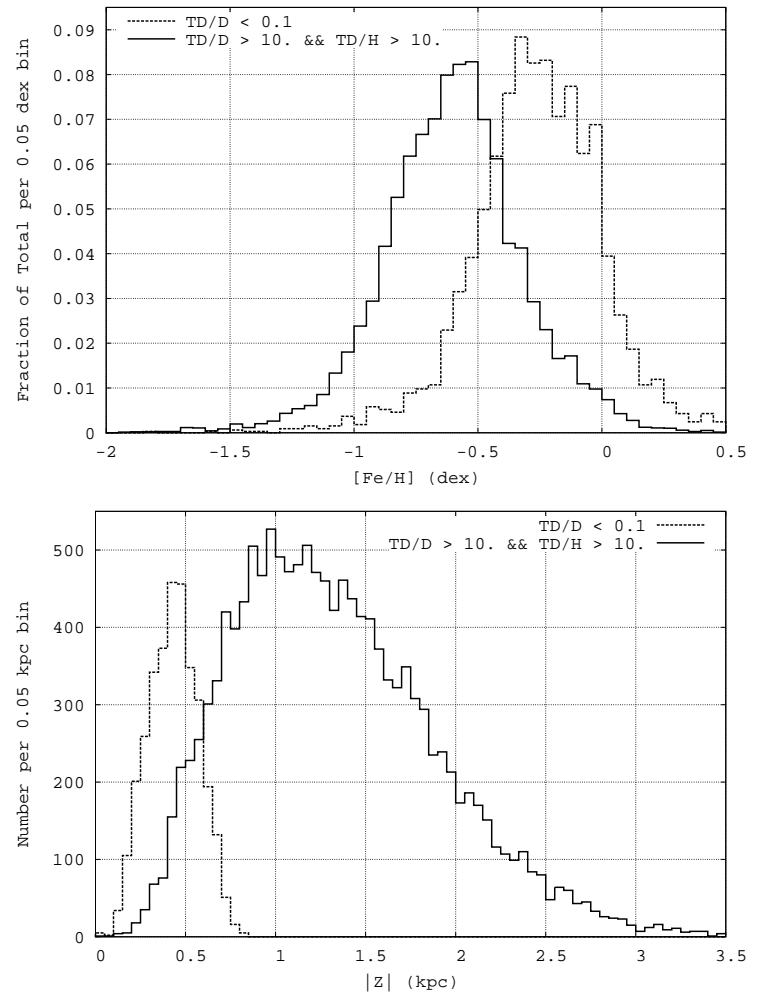

Fig. 4.- Distribution in $|Z|$ (lower) and $[\mathrm{Fe} / \mathrm{H}]$ (upper) for stars selected according to probabilities from spatial and kinematic information as described in the text.

that for vertical heights of $0.5<|Z|<1.0 \mathrm{kpc}$ our dwarf star sample contains both thin and thick disk stars ( $\sim 80 \%$ of the kinematically selected disk stars are thick disk), while above $|Z|=1 \mathrm{kpc}$ we only have thick disk stars.

Using the large sample of dwarf stars with reliably determined properties and a number of previous values with which to compare, we can now provide a better view of the overall abundance patterns of dwarf stars in the Galactic thick disk in both the radial and vertical directions.

\section{Results}

When looking for metallicity gradients of the disk many factors are involved that could affect the outcome. The population of stars, their locations in the Galaxy, and their kinematics are all distinguishing characteristics that can (and do) give different results. In this paper we use exclusively dwarf stars that are mostly from the thick disk component of our Galaxy. We have a very large sample, however, with a wide range of distances and kinematics. As such, we divide our sample based on these properties. Specifically, as discussed in the previous section, we find that for vertical heights of $|Z|>1 \mathrm{kpc}$ our sample has no thin disk contamination and for $0.5<|Z|<1.0$ $\mathrm{kpc}$ the contamination is approximately $20 \%$. Furthermore, we group our stars by their positions in order to disentangle the affect of one metallicity gradient on the other. For example, if we simply took our entire sample and fit for a vertical metallicity gradient using all radial distances, the contribution of a radial metallicity gradient could have an affect on the vertical metallicity gradient. We choose bin sizes of $0.5 \mathrm{kpc}$ in order to have a statistically significant sample of stars in each bin and minimize the affect of the other gradient. We also provide metallicity gradients without binning to see how these values agree with those previously published.

It is important to note that while we do have stars in the full range of fitted values, not all the bins will have the same distribution of radial and/or vertical distances. This could affect our gradients. By averaging the points in each bin, however, and then determining the gradients from these averaged values we reduce the affect of these changes and any potential outliers in our sample.

To estimate errors in the metallicity gradients, a bootstrapping method was employed where we randomly re-sampled half the number of stars in each group one thousand times and fit each one for a gradient. The errors quoted in the tables that follow are the standard deviations of the results from this method. To determine the affect of a systematic difference in the distance estimates we have included metallicity gradients using both the isochrone and photometric distance methods.

\subsection{Radial Gradients}

We first look for metallicity gradients in the radial direction for our sample stars. The presence of a radial metallicity gradient in the Galactic plane has been observed using various methods (blue stars, open clusters, nebulae, etc). A radial metallicity gradient away from the Galactic plane is not as well established. In fact, depend- 
ing on the population and height above the plane, different results are found. Allende Prieto et al. (2006) find no evidence of a radial metallicity gradient for stars in the range $1<|Z|<3 \mathrm{kpc}$ and while Nordström et al. (2004) find a positive gradient for their old age sample (>10 Gyr), their value is still consistent with no gradient within errors. Recent results from RAVE have found positive gradients for likely thick disk stars selected in various ways and their results are consistent with a positive, nonzero gradient within errors. Radial metallicity gradients from various references can be found in Table 2 .

Since we have such a large sample, we are able to look not only for a radial metallicity gradient, but also see how the gradient changes as a function of height above the Galactic plane. In Figure 5 we show metallicity as a function of the radial distance from the Galactic center for our entire sample of stars for different ranges in the vertical height. The resulting radial metallicity gradients can be found in Table 3 and a plot of the radial metallicity gradient as a function of average $|Z|$ is shown in Figure 6. For reference, horizontal lines have been drawn in Figure 6 for the results of previous publications found in Table 2 and two overlapping points in $|Z|$ from Cheng et al. (2012) have been included as well. For vertical heights of $1<|Z|<3 \mathrm{kpc}$ we find that the radial metallicity gradients are relatively constant as a function of $|Z|$. In the vertical height bin where thin disk stars contaminate our sample $(0.5<|Z|<1.0 \mathrm{kpc})$ the radial metallicity gradient is slightly lower. In the highest vertical height bin $(2.5<|Z|<3.0$ $\mathrm{kpc}$ ) the number of stars is an order of magnitude smaller than the bins with lower vertical heights and the difference between the results using the two different distance estimates is largest. Furthermore, from Figure 5] one can see that in this vertical height range the point for radial distances of $10.0<R<10.5 \mathrm{kpc}$ using the isochrone method is noticeably above the fit and forces the linear fit to be steeper. Disregarding this point when fitting for the metallicity gradient gives a value $\left(+0.023 \mathrm{dex} \mathrm{kpc}^{-1}\right)$ that is more consistent with lower heights and the photometric distance estimate. We have included this value in Figure 6 as the open square.

We could fit for a radial metallicity gradient for the entire range associated with the thick disk

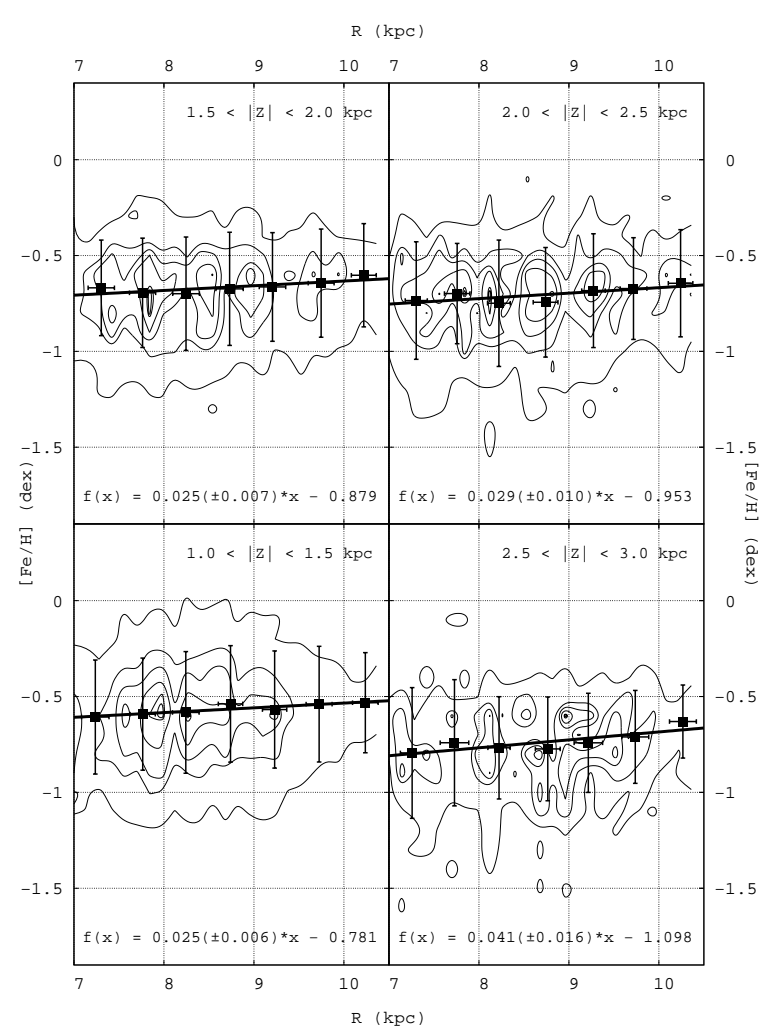

Fig. 5.- Fits for the radial metallicity gradient of the dwarf star sample in different $|Z|$ ranges. Contour lines are shown for 95\%, 90\%, 68\%, 50\%, $33 \%$, and $10 \%$ of the peak density.

$(1<|Z|<3 \mathrm{kpc})$, however, as is evident from the offsets in the linear fits shown in Figure 5] there is a strong vertical metallicity gradient in the sample that will affect an overall determination in the radial direction.

Radial metallicity gradients determined using the photometric method distances are very similar to those found with the isochrone method. Indeed, the values all agree with each other within errors. The fact that there is no large discrepancy between the results using the two methods means that systematic differences in the distances on the order we observe between the two distance estimates (up to $\sim 20 \%$ ) do not significantly affect our results.

Our radial metallicity gradients lie in the middle of the previously published values and agree most closely with those of Nordström et al. (2004) 
Table 2: Radial metallicity gradients from the literature.

\begin{tabular}{cccr}
\hline & gradient $\left(\mathrm{dex} \mathrm{kpc}^{-1}\right)$ & error & notes \\
\hline Allende Prieto et al. (2006) & $\sim 0$ & & $1<|Z|<3 \mathrm{kpc}$ \\
\hline Nordström et al. (2004) & +0.028 & 0.036 & age $>10 \mathrm{Gyr}$ \\
\hline & +0.016 & 0.011 & $\mathrm{~F} \mathrm{dwarfs,} \mathrm{TD} / \mathrm{D}>10$ \\
& +0.016 & 0.012 & $\mathrm{~F} \mathrm{dwarfs,} \mathrm{e}_{v}>0.1$ \\
Coskunoğlu et al. (2012) & +0.010 & 0.009 & G dwarfs, TD $/ \mathrm{D}>10$ \\
& +0.037 & 0.016 & G dwarfs, $\mathrm{e}_{v}>0.1$ \\
\hline Ruchti et al. (2011) & +0.01 & 0.04 & {$[\mathrm{Fe} / \mathrm{H}]<-1.2$} \\
\hline Cheng et al. (2012) & +0.0028 & ${ }_{-0.0052}^{+0.0071}$ & $1<|Z|<1.5 \mathrm{kpc}$ \\
& -0.013 & ${ }_{-0.0016}^{+0.0093}$ & $0.5<|Z|<1.0 \mathrm{kpc}$ \\
\hline
\end{tabular}

Table 3: Radial metallicity gradients of our dwarf star sample.

\begin{tabular}{ccccccccc}
\hline $\begin{array}{c}|Z| \begin{array}{c}\text { Height } \\
(\mathrm{kpc})\end{array} \\
\text { stars }\end{array}$ & $\begin{array}{c}\mathrm{I} \text { (kpc) } \\
(\mathrm{kpc})\end{array}$ & $\begin{array}{c}\bar{R} \\
(\mathrm{kpc})\end{array}$ & $\begin{array}{c}\text { Gradient } \\
\left(\mathrm{dex} \mathrm{kpc}^{-1}\right)\end{array}$ & $\begin{array}{c}N \\
\text { stars }\end{array}$ & $\begin{array}{c}|\bar{Z}| \\
(\mathrm{kpc})\end{array}$ & $\begin{array}{c}\bar{R} \\
(\mathrm{kpc})\end{array}$ & $\begin{array}{c}\text { Gradient } \\
\left(\mathrm{dex} \mathrm{kpc}^{-1}\right)\end{array}$ \\
\hline $0.5-1.0$ & 16,892 & 0.74 & 8.46 & $+0.015 \pm 0.005$ & 15,150 & 0.75 & 8.52 & $+0.017 \pm 0.005$ \\
\hline $1.0-1.5$ & 8,881 & 1.22 & 8.47 & $+0.025 \pm 0.006$ & 9,424 & 1.22 & 8.50 & $+0.019 \pm 0.006$ \\
\hline $1.5-2.0$ & 4,314 & 1.72 & 8.53 & $+0.025 \pm 0.007$ & 4,954 & 1.72 & 8.56 & $+0.021 \pm 0.007$ \\
\hline $2.0-2.5$ & 1,907 & 2.22 & 8.59 & $+0.029 \pm 0.010$ & 2,519 & 2.22 & 8.56 & $+0.027 \pm 0.008$ \\
\hline $2.5-3.0$ & 730 & 2.71 & 8.68 & $+0.041 \pm 0.016^{\text {a }}$ & 1,136 & 2.72 & 8.64 & $+0.022 \pm 0.014$ \\
\hline
\end{tabular}

${ }^{a}$ Excluding the point with radial distances of $10.0<R<10.5 \mathrm{kpc}$ gives a metallicity gradient of $+0.023 \mathrm{dex} \mathrm{kpc}^{-1}$.

and the F dwarf samples of Coskunoğlu et al. (2012). Our results differ from those of Cheng et al. (2012) in the bins that overlap with their work. As is shown in Figure 2 there is a noticeable change in the metallicity distribution for stars with surface gravities below $\sim 4.4$ dex in our sample, which could be the reason for the discrepancy between our work using high surface gravity dwarfs and their work using turnoff stars with lower surface gravities. It is also possible that there are different ratios of thin and thick disk stars between our sample and theirs, which would obviously give a different metallicity gradient if the two populations themselves had gradients different from each other.

\subsection{Vertical Gradients}

In a similar fashion to what was done for the radial gradients, we examine the vertical metallicity gradients of our sample. In Table 4 we list some previously published values using various populations and sample selection methods.
We bin in radial distance to fit for vertical metallicity gradients and restrict our fits to vertical heights of $1<|Z|<3 \mathrm{kpc}$ for two reasons. The first is to be more consistent with previously published values. The second is to provide a clearer picture of the vertical metallicity gradient of only the thick disk. There is a significant overlap of the thin and thick disks for vertical heights of $|Z|<1 \mathrm{kpc}$, which can be seen in the vertical distribution of the kinematically separated samples shown in Figure 4. Therefore, if we extend our fits below this height for our full sample of stars we will not be probing a single population of our Galaxy, but the contribution from both the thin and thick disks. As we will show, the kinematically selected thick disk with $|Z|<1 \mathrm{kpc}$ could be included in the fits without significantly altering the results, but in order to be more consistent with previous results we use only vertical heights of $1<|Z|<3 \mathrm{kpc}$.

Vertical metallicity gradients for our sample of stars are given in Table 5. Plots showing the verti- 
Table 4: Vertical metallicity gradients from the literature.

\begin{tabular}{cccr}
\hline & gradient $\left(\mathrm{dex} \mathrm{kpc}^{-1}\right)$ & error & notes \\
\hline Allende Prieto et al. (2006) & $\sim 0$ & & $1<|Z|<3 \mathrm{kpc}$ \\
\hline Chen et al. (2011) & -0.120 & 0.01 & Galaxy model and fits \\
& -0.225 & 0.07 & All points \\
\hline Katz et al. (2011) & -0.068 & 0.009 & 2 lines of sight \\
\hline Ruchti et al. (2011) & -0.09 & 0.05 & {$[\mathrm{Fe} / \mathrm{H}]<-1.2$} \\
\hline Kordopatis et al. (2011) & -0.14 & 0.05 & $1<|Z|<4 \mathrm{kpc}$ \\
\hline
\end{tabular}

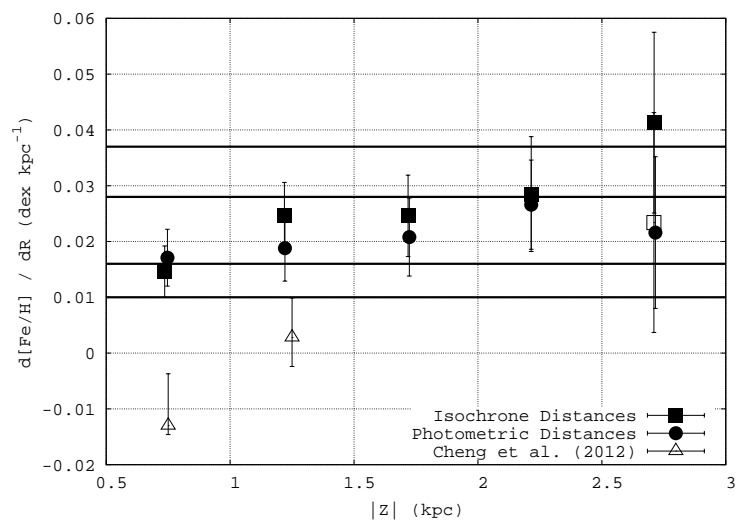

Fig. 6.- Radial metallicity gradients of our dwarf star sample found using distances from the isochrone (solid squares) and photometric (solid circles) methods for different heights above the Galactic plane. The horizontal lines and open triangles correspond to previously published values (see Table 2).

cal metallicity gradients in different radial bins can be found in Figure 7. This plot shows the effect of selecting only stars with heights above $|Z|=1 \mathrm{kpc}$. The points in the $0.5<|Z|<1.0 \mathrm{kpc}$ bin show the average metallicity of the full sample (open squares) and of the kinematically selected thick disk sample (open circles). Careful examination of these points shows that the full sample has a higher metallicity and is systematically above the fit value. This is most certainly due to the contribution of the more metal-rich thin disk in our sample at these heights and is the reason we have excluded this region from our metallicity gradient determinations. The kinematically selected thick disk sample matches well with the fit value.

A plot of the vertical metallicity gradients as a function of radial distance from the Galactic center is found in Figure 8. It is obvious that the metallicity gradient for the largest radial distances are the most discrepant between the two distance determinations. As was noted for the radial metallicity gradients, these distances have the lowest number of stars making statistics a possible issue in the metallicity gradient determination. From Figure 7 one can see that including the $0.5<|Z|<1.0 \mathrm{kpc}$ bin in the fit for radial distances of $10.0<R<10.5 \mathrm{kpc}$ found using the isochrone method would give a steeper slope. This would bring the result closer to the values at smaller Galactic radii as well as the result using photometric distances. As was stated above, however, for consistency with previous results we have restricted our fits to vertical heights of $1<|Z|<3$ kpc.

There is some structure to the gradients as a function of radial distance but no obvious trends. In fact, a single value can describe our results. Because the radial metallicity gradient is much smaller (by an order of magnitude) than the vertical metallicity gradient, we can fit the data for stars with radial distances of $7.0<R<10.5 \mathrm{kpc}$ and vertical heights of $1<|Z|<3 \mathrm{kpc}$, which gives us values of $-0.113 \pm 0.010$ and $-0.125 \pm 0.008$ dex $\mathrm{kpc}^{-1}$ for our full dwarf star sample using isochrone and photometric distances respectively. These values are in very good agreement with the shallower vertical metallicity gradient found by Chen et al. (2011) as well as those given in Kordopatis et al. (2011) and Ruchti et al. (2011). It is slightly steeper than the result of Katz et al. (2011).

Vertical metallicity gradients found using the photometric distance determination are very similar to those found using the isochrone distance determination. The values from each method agree within errors and there is no obvious systematic 
Table 5: Vertical metallicity gradients of our dwarf star sample with heights of $1<|Z|<3 \mathrm{kpc}$.

\begin{tabular}{|c|c|c|c|c|c|c|c|c|}
\hline \multirow[b]{2}{*}{$\begin{array}{c}R \text { Distance } \\
(\mathrm{kpc})\end{array}$} & \multicolumn{4}{|c|}{ Isochrone Distance Method } & \multicolumn{4}{|c|}{ Photometric Distance Method } \\
\hline & $\begin{array}{c}N \\
\text { stars }\end{array}$ & $\begin{array}{c}\overline{|Z|} \\
(\mathrm{kpc})\end{array}$ & $\begin{array}{c}\bar{R} \\
(\mathrm{kpc})\end{array}$ & $\begin{array}{c}\text { Gradient } \\
\left(\operatorname{dex~kpc}^{-1}\right)\end{array}$ & $\begin{array}{c}N \\
\text { stars }\end{array}$ & $\begin{array}{c}\overline{|Z|} \\
(\mathrm{kpc})\end{array}$ & $\begin{array}{c}\bar{R} \\
(\mathrm{kpc})\end{array}$ & $\begin{array}{c}\text { Gradient } \\
\left(\operatorname{dex~} \mathrm{kpc}^{-1}\right)\end{array}$ \\
\hline $7.0-7.5$ & 1,812 & 1.58 & 7.27 & $-0.13 \pm 0.03$ & 2,042 & 1.67 & 7.26 & $-0.12 \pm 0.03$ \\
\hline $7.5-8.0$ & 3,125 & 1.52 & 7.76 & $-0.09 \pm 0.03$ & 3,473 & 1.57 & 7.76 & $-0.12 \pm 0.02$ \\
\hline $8.0-8.5$ & 3,427 & 1.48 & 8.24 & $-0.12 \pm 0.02$ & 3,827 & 1.52 & 8.25 & $-0.13 \pm 0.02$ \\
\hline $8.5-9.0$ & 2,913 & 1.54 & 8.74 & $-0.16 \pm 0.02$ & 3,286 & 1.57 & 8.74 & $-0.16 \pm 0.02$ \\
\hline $9.0-9.5$ & 2,296 & 1.55 & 9.23 & $-0.11 \pm 0.02$ & 2,674 & 1.59 & 9.23 & $-0.13 \pm 0.02$ \\
\hline $9.5-10.0$ & 1,423 & 1.65 & 9.73 & $-0.11 \pm 0.02$ & 1,684 & 1.67 & 9.73 & $-0.09 \pm 0.02$ \\
\hline $10.0-10.5$ & 836 & 1.67 & 10.24 & $-0.07 \pm 0.03$ & 1,047 & 1.74 & 10.24 & $-0.13 \pm 0.03$ \\
\hline $7.0-10.5$ & 15,832 & 1.54 & 8.51 & $-0.113 \pm 0.010$ & 18,033 & 1.59 & 8.53 & $-0.125 \pm 0.008$ \\
\hline
\end{tabular}

difference between the two. The only discrepancy between the metallicity gradients determined using the different distance estimates is for the bin with $10.0<R<10.5 \mathrm{kpc}$, which also has the lowest statistics.

\section{Discussion}

We have probed the metallicity gradients in both the radial and vertical directions using dwarf stars selected from SDSS. We kinematically separate this sample into thin and thick disk components at vertical heights where the two populations are known to overlap using probabilities defined and used in previous analyses. This is significant because this separation is most often applied to stars in the solar neighborhood, but we have shown that it can be successfully used for SDSS data with vertical heights of up to $1 \mathrm{kpc}$ by taking into account the local number density as a function of height.

Stars associated with the Galactic thick disk have a positive radial metallicity gradient for heights of $1<|Z|<3 \mathrm{kpc}$. Our results seem to be at odds with the results of Cheng et al. (2012) in the two vertical height bins that overlap. However, the different populations and selection methods between our work and theirs make it difficult to compare the two results directly. Our results indicate that a radial metallicity gradient determined for stars with $|Z|<1.0 \mathrm{kpc}$ will heavily depend on the fraction of thin and thick disk stars in the sample if the two populations have very distinct values and trends.
When fitting the entire sample of stars with vertical distances most consistent with the thick disk, we find a radial metallicity gradient that is positive and not consistent with zero within errors. In particular, we find radial metallicity gradients of $+0.025 \pm 0.006(+0.019 \pm 0.006),+0.025$ $\pm 0.007(+0.021 \pm 0.007),+0.029 \pm 0.010(+0.027$ $\pm 0.008)$, and $+0.041 \pm 0.016(+0.022 \pm 0.014)$ dex $\mathrm{kpc}^{-1}$ using isochrone (photometric) distances for stars in $0.5 \mathrm{kpc}$ bins in vertical height between $1<|Z|<3 \mathrm{kpc}$ and with radial distances of $7.0<R<10.5 \mathrm{kpc}$. Restricting the fit for the highest vertical height bin to $7.0<R<10.0 \mathrm{kpc}$ changes the result using isochrone distances from

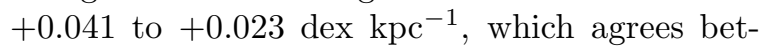
ter with lower heights and with the photometric distance determination. To our knowledge, this is the first time that a radial metallicity gradient for the thick disk has been found at these vertical heights. The values we find are in very good agreement with the old stellar sample value of Nordström et al. (2004) and with both of the thick disk F dwarf samples of Coşkunoğlu et al. (2012). It also agrees within errors with the results of Ruchti et al. (2011). Due to the size of our sample, our statistical error is smaller than these previous works.

Fitting the full sample of stars with radial distances of $7.0<R<10.5 \mathrm{kpc}$ in the range $1<$ $|Z|<3 \mathrm{kpc}$, we find a vertical metallicity gradient of $-0.113 \pm 0.010(-0.125 \pm 0.008)$ dex kpc $\mathrm{kp}^{-1}$ using isochrone (photometric) distances. This value is in good agreement with the results of Chen et al. (2011), Kordopatis et al. (2011), and 


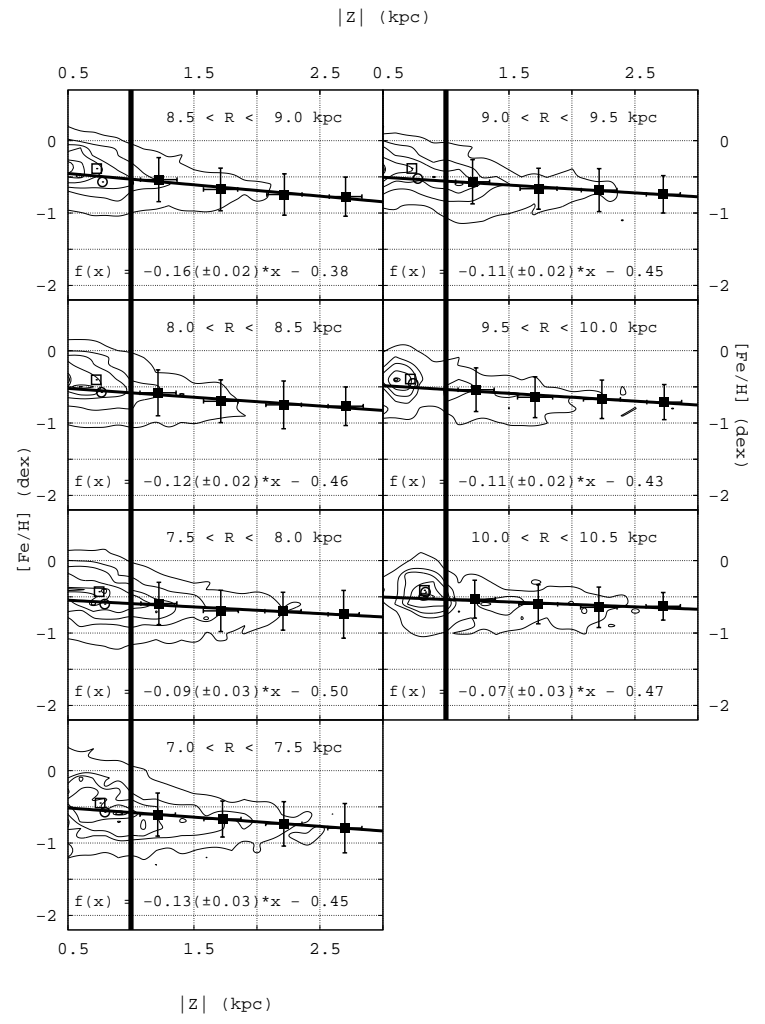

Fig. 7.- Fits for the vertical metallicity gradient of the dwarf star sample in different $R$ ranges. Contour lines are shown for $95 \%, 90 \%, 68 \%, 50 \%$, $33 \%$, and $10 \%$ of the peak density.

\section{Ruchti et al. (2011).}

The results of Bovy et al. (2012) (among others) suggests that the Galactic disk is a single component that changes smoothly as a function of both radial and vertical distances. We observe no obvious or significant trends in either the radial or vertical metallicity gradients for stars most consistent with the thick disk of our Galaxy. However, it is not clear if the kinematic selection, distances, and/or bin sizes we have used would be able to detect changes in the metallicity gradient due to such a scenario. Furthermore, as discussed in their work, dividing the disk into components using different methods can give conflicting results, making it difficult to compare the results from geometric, kinematic, and abundance decompositions.

In summary, the metallicity gradients we find in both the radial and vertical directions using the

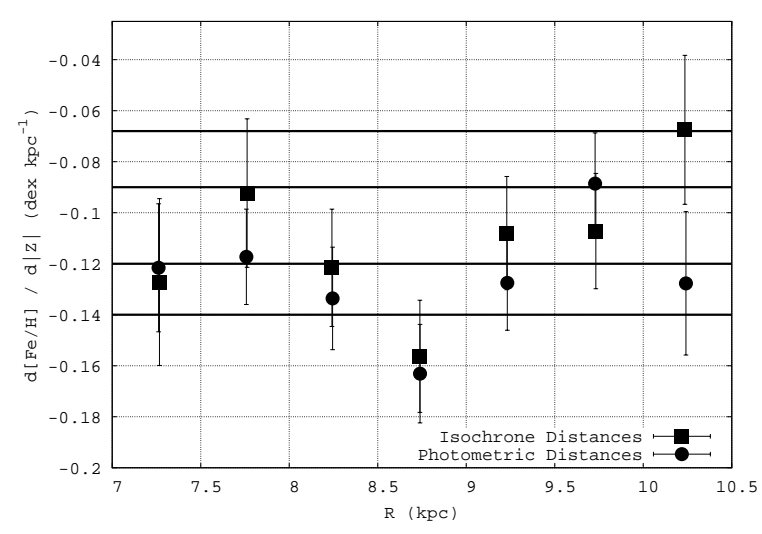

Fig. 8.- Vertical metallicity gradients of our dwarf star sample found using distances from the isochrone (solid squares) and photometric (solid circles) methods for different radial distances from the Galactic center. The horizontal lines correspond to previously published values (see Table 4).

sample of dwarf stars selected from SDSS DR8 show no large or obvious trends in the distance ranges we are able to probe. The vertical metallicity gradient we have found is much steeper than the radial metallicity gradient, but we have found both to be nonzero. Future large spectroscopic survey programs, such as LAMOST (Zhao et al. 2006), should provide greater statistics and more complete samples of stars in a wider range of distances so that metallicity gradients can be probed in more detail and an even better understanding of our Galactic disk can be realized.

We would like to thank the anonymous referee for their helpful comments and suggestions for improving the paper. We would also like to thank Dr. Martin Smith and Dr. Ron Wilhelm for their comments and suggestions.

Funding was provided by the National Natural Science Foundation of China under grants 11150110135,11073026 and 11222326 and the Chinese Academy of Sciences under grant KJCX2YW-T22 and the fellowship for young international scientists.

Funding for SDSS-III has been provided by the Alfred P. Sloan Foundation, the Participating Institutions, the National Science Foundation, and the U.S. Department of Energy Office of Science. 
The SDSS-III web site is http://www.sdss3.org/.

SDSS-III is managed by the Astrophysical Research Consortium for the Participating Institutions of the SDSS-III Collaboration including the University of Arizona, the Brazilian Participation Group, Brookhaven National Laboratory, University of Cambridge, University of Florida, the French Participation Group, the German Participation Group, the Instituto de Astrofisica de Canarias, the Michigan State/Notre Dame/JINA Participation Group, Johns Hopkins University, Lawrence Berkeley National Laboratory, Max Planck Institute for Astrophysics, New Mexico State University, New York University, Ohio State University, Pennsylvania State University, University of Portsmouth, Princeton University, the Spanish Participation Group, University of Tokyo, University of Utah, Vanderbilt University, University of Virginia, University of Washington, and Yale University.

\section{REFERENCES}

Abadi, M.G., Navarro, J.F., Steinmetz, M., \& Eke, V.R. 2003, ApJ, 597, 21

Aihara, H., Allende Prieto, C., \& An, D., et al. 2011, ApJS, 193, 29

Allende Prieto, C., Beers, T.C, Wilhelm, R., et al. 2006, ApJ, 636, 804

Bensby, T., Feltzing, S., \& Lundström, I. 2003, A\&A, 410, 527

Bovy, J., Rix, H.-W., Liu, C., et al. 2012, ApJ, 753,148

Brook, C.B., Kawata, D., Gibson, B.K., \& Freeman, K.C. 2004, ApJ, 612, 894

Chen, Y.Q., Zhao, G., Carrell, K., \& Zhao, J.K. 2011, AJ, 142, 184

Cheng, J.Y., Rockosi, C.M., Morrison, H.L., et al. 2012, ApJ, 746, 149

Chiba, M. \& Beers, T.C. 2000, AJ, 119, 2843

Coşkunoğlu, B., Ak, S., Bilir, S., et al. 2012, MNRAS, 419, 2844

Gilmore, G. \& Reid, N. 1983, MNRAS, 202, 1025
Girardi, L., Grebel, E.K., Odenkirchen, M., \& Chiosi, C. 2004, A\&A, 422, 205

Ivezić, Ž., Sesar, B., Jurić, M., et al. 2008, ApJ, 684,287

Johnson, D.R.H. \& Soderblom, D.R. 1987, AJ, 93, 864

Jurić, M., Ivezić, Ž, Brooks, A., et al. 2008, ApJ, 673,864

Katz, D., Soubiran, C., Cayrel, R., et al. 2011, A\&A, 525, A90

Kordopatis, G., Recio-Blanco, A., de Laverny, P., et al. 2011, A\&A, 535, A107

Nordström, B., Mayor, M., Anderson, J., et al. 2004, A\&A, 418, 989

Ruchti, G.R., Fullbright, J.P., Wyse, R.F.G., et al. 2011, ApJ, 737, 9

Schlesinger, K.J., Johnson, J.A., Rockosi, C.M., et al. 2011, arXiv:1112.2214v1 (submitted to ApJ)

Schönrich, R. \& Binney, J. 2009, MNRAS, 399, 1145

Schönrich, R., Binney, J., \& Dehnen, W. 2010, MNRAS, 403, 1829

Soubiran, C., Bienaymé, O., \& Siebert, A. 2003, A\&A, 398, 141

Steinmetz, M., Zwitter, T., Siebert, A., et al. 2006, AJ, 132,1645

Zhao, G., Chen, Y.Q., Shi, J.R., et al. 2006, Chinese J. Astron. Astrophys., 6, 265

Zwitter, T., Matijevič, G., Breddels, M.A., et al. 2010, A\&A, 522, A54

This 2-column preprint was prepared with the AAS LATEX macros $\mathrm{v} 5.2$. 